\title{
A Case of Adult Onset Still's Disease Misdiagnosed as Septic Arthritis
}

\author{
Sang Jun Song, $\mathrm{MD}, \mathrm{PhD}^{1}$, Dae Kyung Bae, $\mathrm{MD}, \mathrm{PhD}^{1}$, Jung Ho Noh, MD, $\mathrm{PhD}^{2}$, Geon Wook Seo, $\mathrm{MD}^{1}$ and \\ Dong Cheol Nam, MD ${ }^{1}$ \\ Department of Orthopaedic Surgery, ${ }^{1}$ Kyung Hee University College of Medicine, ${ }^{2}$ National Police Hospital, Seoul, Korea
}

\begin{abstract}
We present a case of adult onset Still's disease (AOSD) that was misdiagnosed as septic arthritis of the shoulder and knee. A forty-nine-year-old woman was admitted for pain in the left knee. The patient's medical history showed that she had undergone arthroscopic irrigation twice and an open debridement under the diagnosis of septic shoulder at another hospital. The laboratory and joint fluid analysis findings led us to suspect septic knee. Arthroscopic irrigation and antibiotics treatment were performed. At five weeks after discharge, she presented with pain in the same joint, fever, and rash. The symptoms were consistent with Yamaguchi's criteria for AOSD. We started corticosteroid therapy, and clinical remission was achieved. In conclusion, we suggest that AOSD should be considered as a diagnosis of exclusion to avoid misdiagnosis with septic arthritis.
\end{abstract}

Key words: Knee, Adult onset Still's disease, Septic arthritis.

Adult onset Still's disease (AOSD) is a rare inflammatory disorder of unknown etiology. The reported prevalence ranges from 1 to 10 cases per million ${ }^{1)}$. AOSD is difficult to suspect for orthopedic surgeon because of its low prevalence and lack of awareness. The diagnosis of AOSD is difficult and is made based on Yamaguchi's criteria ${ }^{2}$ after exclusion of infectious diseases, malignancies or autoimmune diseases. Three types of disease progression have been described ${ }^{1,3)}$. The monocyclic type is characterized by systemic symptoms of fever, rash, organomegaly, and serositis. The polycyclic type is characterized by multiple recurrences that may or may not affect the joints. Complete remission is usually achieved between flares that may be separated by many years. The chronic articular type primarily

Received April 26, 2011; Revised June 7, 2011; Accepted July 4, 2011. Correspondence to: Dae Kyung Bae, MD.

Department of Orthopaedic Surgery, Kyung Hee University College of Medicine, \#1 Hoeki-dong, Dongdaemun-gu, Seoul 130-702, Korea.

Tel: +82-2-958-8366, Fax: +82-2-962-9817

Email: bdkyung@khmc.or.kr

This is an Open Access article distributed under the terms of the Creative Common Attribution Non-Commercial License (http://creativecommons.org/licenses/by-nc/3.0/) which permits unrestricted non-commercial use, distribution, and reproduction in any medium, provided the original work is properly cited.

Copyright @ 2011. THE KOREAN KNEE SOCIETY

www.jksrr.org targets the joints. Severe joint destruction is often the end result in untreated cases. Especially, the chronic articular type AOSD has been often mistaken for septic arthritis because there are minimal to no systemic symptoms in the early stage of the disease.

We encountered a case that was mistaken for septic shoulder at another hospital and for septic knee at our hospital during the disease progression. Unnecessary surgical and antibiotic treatments were performed repetitively. Here we report the case of chronic articular type AOSD that was misdiagnosed as septic arthritis with a review of the literature that emphasizes the clinical and laboratory characteristics of articular manifestations.

\section{Case Report}

A forty-nine-year-old woman was admitted to our orthopedic department for pain in the left knee that had lasted for two weeks. The patient's medical history revealed that she had been admitted to another hospital for pain in the right shoulder five months ago. During admission to another hospital, she had high spiking fever and skin rash. But, those clinical findings were considered drug fever and eruption. Arthroscopic irrigation was performed twice and open debridement was carried out once in the right shoulder under the diagnosis of refractory septic shoulder. Prolonged intravenous antibiotic treatment had been administrated for two months due to persistent fever, signs of infections in the right 
Table 1. Laboratory Data During the Course of Treatment

\begin{tabular}{|c|c|c|c|c|}
\hline Component & Another hospital & 1st admission & 2nd admission & After prednisolon \\
\hline WBC (k/UI) & 17,600 & 30,220 & 15,460 & 8,600 \\
\hline Neutrophils (\%) & 87 & 91.2 & 88.9 & 54.1 \\
\hline Hemoglobin (g/dL) & 10.2 & 10.0 & 9.1 & 10.7 \\
\hline Hematocrit (\%) & 30.5 & 30.6 & 27.3 & 29.8 \\
\hline Platelet (k/uL) & 487 & 381 & 527 & 539 \\
\hline $\operatorname{ESR}(\mathrm{mm} / \mathrm{h})$ & 78 & 81 & 83 & 2 \\
\hline C-reactive protein $(\mathrm{mg} / \mathrm{dL})$ & 19.47 & 18.2 & 13.2 & 0.23 \\
\hline Total protein $(\mathrm{g} / \mathrm{dL})$ & 7.5 & 7.5 & 7.2 & 6.1 \\
\hline Albumin (g/dL) & 3.7 & 3.2 & 3.6 & 4.1 \\
\hline Bilirubin, direct (mg/dL) & - & 0.2 & 0.26 & - \\
\hline Bilirubin total (g/dL) & 0.4 & 0.71 & 0.64 & 0.64 \\
\hline $\operatorname{ALP}(\mathrm{IU} / \mathrm{L})$ & 575 & 133 & 97 & - \\
\hline AST (IU/L) & 129 & 30 & 24 & 11 \\
\hline ALT (IU/L) & 42 & 22 & 13 & 10 \\
\hline INR & 1.03 & 1.11 & 1.10 & - \\
\hline Prothrombin time (s) & 13.4 & 14.4 & 14.3 & - \\
\hline Ferritin (ng/mL) & - & - & 593.5 & 53.9 \\
\hline RF & - & $<10$ & - & - \\
\hline HLA B27 & - & - & - & - \\
\hline ANA & - & - & Non-reactive & - \\
\hline
\end{tabular}

WBC: white blood cell, ESR: erythrocyte sedimentation rate, ALP: alkaline phosphatase, ALT: alanine amino transaminase, AST: aspartate aminotransferase, INR: international normalized ratio, RF: rheumatoid factor, HLA: human leukocyte antigen, ANA: antinuclear antibody.

shoulder, and the fluctuation in the serologic test values including the white blood cell (WBC) count, erythrocyte sedimentation rate (ESR), and C-reactive protein (CRP) (Table 1). Eventually, the fever had dropped and the pain in the right shoulder had been improved slightly. She had been discharged from that hospital after two months, but pain and effusion developed in the left knee 3 months later.

During admission at our hospital, there were no fever and skin rash (Fig. 1). The initial laboratory test revealed WBC counts, 30,220 cells $/ \mathrm{mm}^{3}$ with a neutrophillic predominance $(91.2 \%)$; hemoglobin, $10.0 \mathrm{~g} / \mathrm{dL}$; platelets, $381 \mathrm{~K} / \mathrm{mm}^{3}$; ESR, $81 \mathrm{~mm} / \mathrm{hr}$; CRP, $18.2 \mathrm{mg} / \mathrm{dL}$; aspartate aminotransferase (AST), $30 \mathrm{U} / \mathrm{L}$; alanine amino transaminase (ALT), $22 \mathrm{U} / \mathrm{L}$; rheumatoid factor, $<10 \mathrm{IU} / \mathrm{mL}$; and negative human leukocyte antigen (HLA) B-27 (Table 1). Radiographs of the knee showed no significant abnormal findings (Fig. 2). MRI of the left knee showed synovial hypertrophy and joint effusion, which led us to suspect low virulent infectious arthritis (Fig. 3). The aspirated fluid from the knee joint was analyzed with the suspicion of septic knee. The analysis showed a WBC count of $52,000 / \mathrm{mm}^{3}$, neutrophil of $94 \%$,

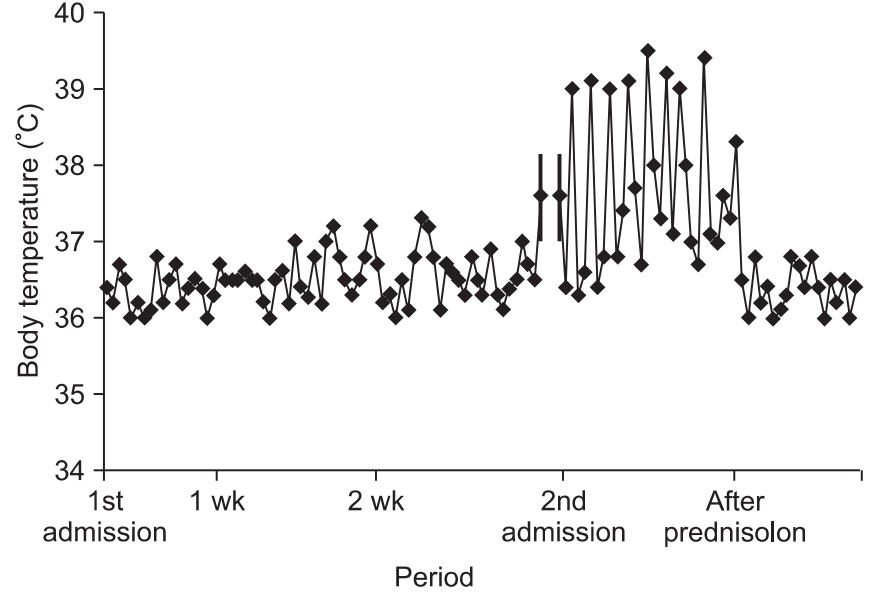

Fig. 1. Patterns of fever during the first and second admission. There was no fever during the first admission, but there was high spiking fever during the second admission.

and negative crystal. We performed an arthroscopic irrigation and prescribed intravenous antibiotics for 3 weeks. Thereafter, the symptoms of the left knee joint were improved, and the patient 


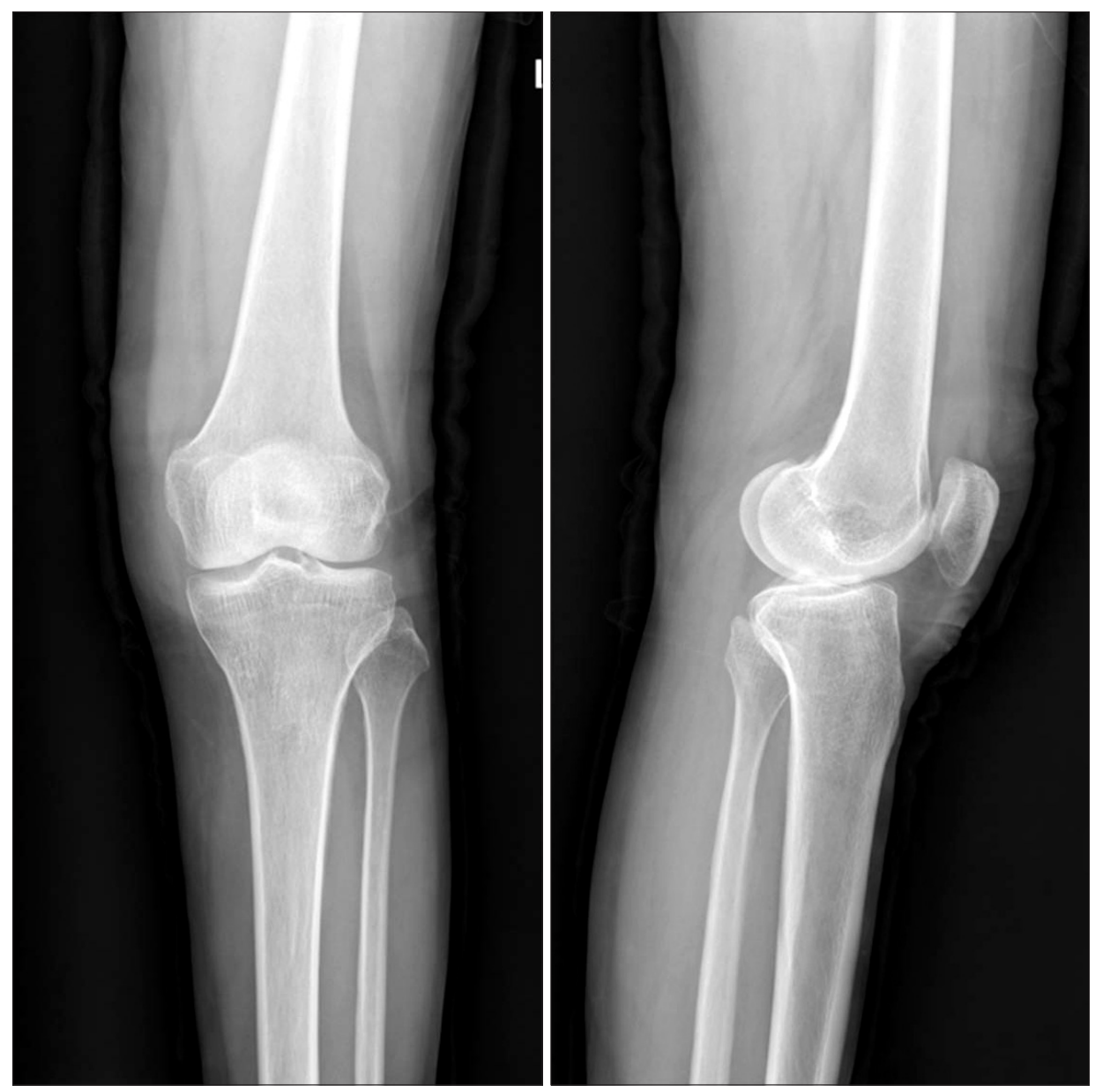

Fig. 2. Radiographs of the knee in adult onset Still's disease. Anteroposterior and lateral views of the knee showed no significant abnormal findings.
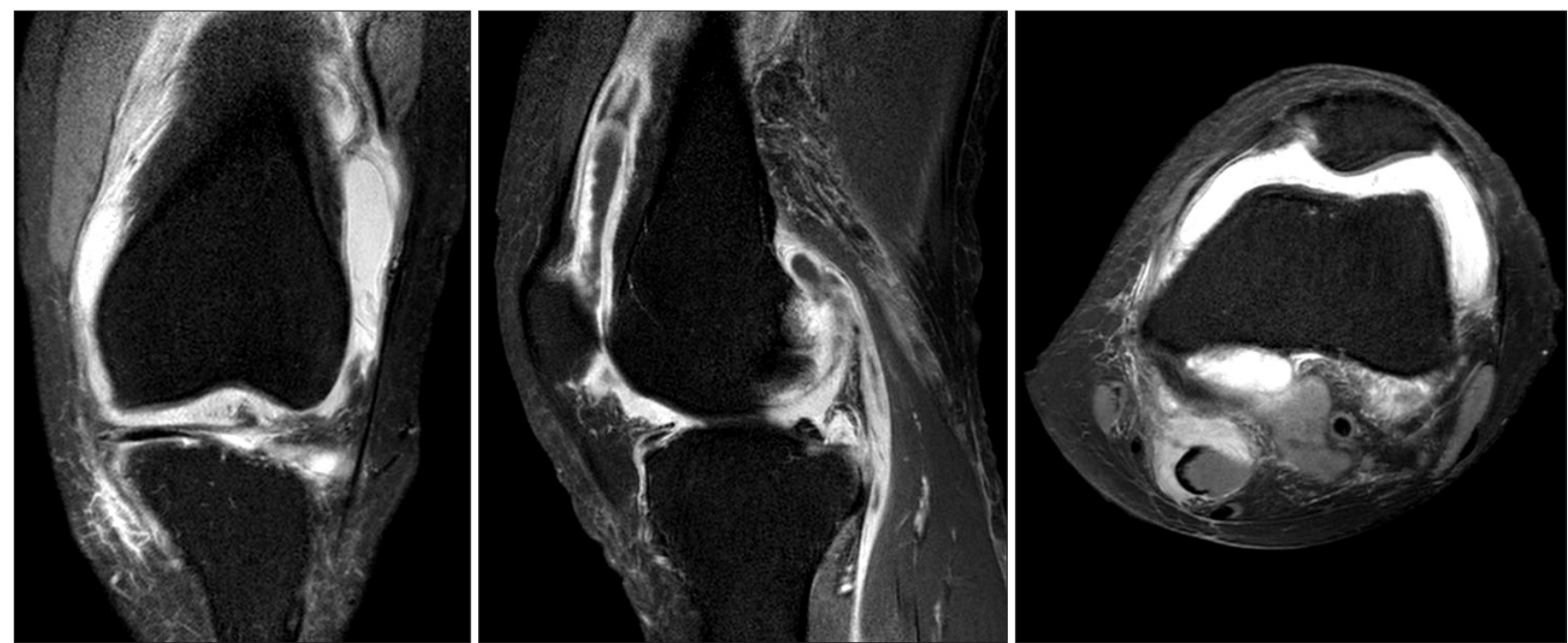

Fig. 3. MRI of the knee in adult onset Still's disease. T2 weighted images showed inflammatory arthritis with the effusion of the knee joint. 
Table 2. Criteria for Adult Onset Still's Disease by Yamaguchi et al. ${ }^{2)}$

Major criteria
Fever $\geq 39^{\circ} \mathrm{C}$ lasting 1 week or more
Arthralgia lasting 2 weeks or more
Typical skin rash: maculopapular, non-pruritic ${ }^{\mathrm{a})}$, salmon-pink rash
$\quad$ with concomitant fever spikes
Leukocytosis $\geq 10,000 / \mathrm{mm}^{3}$ with neutrophil, polymorphonuclear
count $\geq 80 \%$
Minor criteria
Pharyngitis or sore throat
Lymphadenopathy and/or splenomegaly ${ }^{\mathrm{b})}$
Liver enzyme abnormalities (aminotransferases)
Negative for rheumatoid factor or antinuclear antibodies ${ }^{\mathrm{d})}$
Exclusion criteria

Absence of infection, especially sepsis and Epstein-Barr viral infection Absence of malignant disease, especially lymphomas

Absence of inflammatory disease, especially polyarteritis nodosa

Classification of adult Still's disease requires 5 or more criteria including 2 or more major criteria ${ }^{\mathrm{e}}$.

${ }^{a)}$ Macular or maculopapular nonpruritic salmon-pink eruption usually appearing during fever. ${ }^{b}$ Lymphadenopathy is defined as recent development of significant lymph node swelling, and splenomegaly

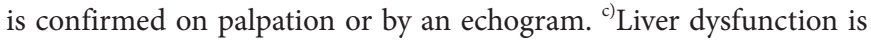
defined as an abnormally elevated level of transaminases and/or lactate dehydrogenase, which is attributed to liver damage associated with this disease but not with drug allergy/toxicity or other causes. For the differentiation, it is recommended to see if liver function returns to normal upon discontinuation of hepatotoxic drug or not, before applying this criterion. ${ }^{\mathrm{d})}$ Rheumatoid factor in serum must be negative by routine test for the detection of IgM-RF, and serum Antinuclear antibody must be negative by routine immunofluorescence test. ${ }^{\text {e) }}$ All criteria are applicable only in absence of other clinical explanations.

was discharged from our hospital.

Five weeks later, the patient presented to the emergency department with left knee joint pain, fever, and rash. At the second admission, body temperature continued to rise to $39^{\circ} \mathrm{C}$. The pattern of fever was quotidian or double-quotidian, peaking in the late afternoon (Fig. 1). A faint pink macular rash appeared on the abdomen, flanks, thighs, and arms. Laboratory test revealed findings similar with those at the first admission. We found that the patient's current symptoms were consistent with Yamaguchi's criteria for AOSD (Table 2). We checked her ferritin level, which was elevated at $593.5 \mathrm{ng} / \mathrm{mL}$. After a consultation with rheumatologists, we confirmed AOSD and started the patient on intravenous prednisolone $60 \mathrm{mg}$ per day for one week. This resulted in a prompt resolution of her fever and rash within 24 hours. Oral administration of the same dose of prednisolone

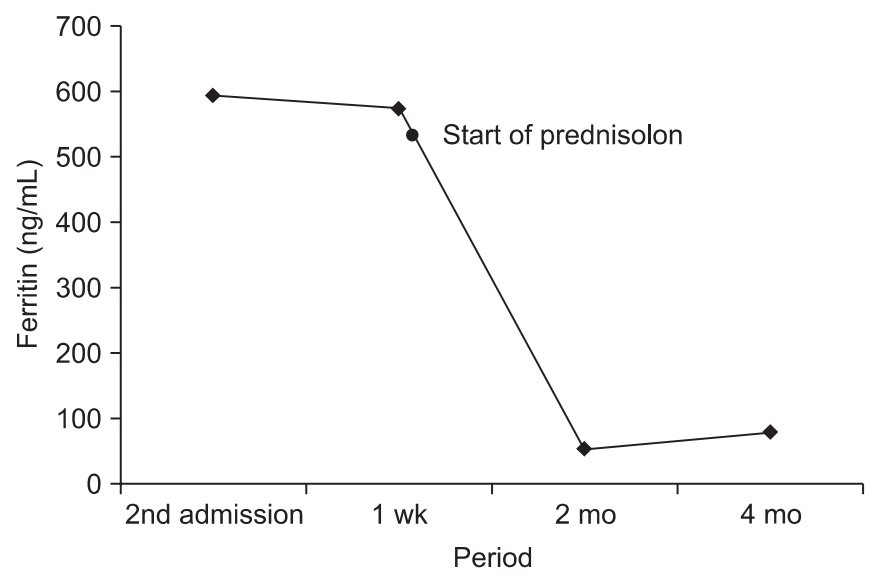

Fig. 4. Change of serum ferritin level after treatment of adult onset Still's disease. The high level of ferritin at diagnosis was normalized after the corticosteroid therapy.

was used for the next two weeks after discharge. Then, the dosage was reduced gradually over 4 months. Nabumetone and hydroxychloroquine sulfate were also prescribed for 4 months along with prednisolone. Now, two years after the first admission at our hospital, the patient is still in remission. Laboratory test revealed WBC counts, 8,600 cells $/ \mathrm{mm}^{3}$; neutrophils percent, $54.1 \%$; ESR, $2 \mathrm{~mm} / \mathrm{h}$; CRP, $0.23 \mathrm{mg} / \mathrm{dL}$; and ferritin, $53.9 \mathrm{ng} / \mathrm{mL}$ (Table 1, Fig. 4).

\section{Discussion}

Still ${ }^{4)}$ in 1896 first recognized Still's disease in children characterized by the triad of quotidian fever, evanescent rash, and arthritis, which has become known as juvenile inflammatory arthritis. AOSD was first described by Bywaters ${ }^{5}$ in 1971 for adults who presented with symptoms indistinguishable from those of the classic childhood Still's disease. The diagnosis of AOSD is based on clinical findings. It requires compliance with Yamaguchi's criteria ${ }^{2)}$, which have a sensitivity of $96.2 \%$ and a specificity of $92.1 \%$. Major criteria include fever, arthralgia, rash, and leukocytosis. Minor criteria include sore throat, lymphadenopathy and/or splenomegaly, and liver dysfunction (Table 2). More recently, a new set of classification criteria that shows a higher specificity and considers glycosylated ferritin has been proposed ${ }^{1,6)}$. Our patient satisfied 4 major and 2 minor no exclusion diagnostic criteria of Yamaguchi, and satisfied 4 major and 2 minor diagnostic criteria of Fautrel according to the clinical manifestations ${ }^{1}$. She was diagnosed with AOSD according to the Yamaguchi and Fautrel diagnostic criteria.

The fever is quotidian or double-quotidian by nature ${ }^{77}$. Typically, it starts suddenly and the temperature rapidly reaches $39^{\circ} \mathrm{C}$ or 
more. It evolves with daily spikes, frequently in the evening, for at least a week. The typical rash is often described as evanescent, salmon to pink colored, maculopapular lesion. It is mainly visible during fever spikes in the proximal limbs and trunk sparing the face ${ }^{8)}$. AOSD is frequently misdiagnosed as a drug allergy to non-steroidal anti-inflammatory drugs (NSAIDs) or antibiotics prescribed for joint symptoms or fever. Fever and rash were noted in our patient during admission at another hospital, but these clinical findings were interpreted as drug fever and eruption. Unfortunately, there was no fever and rash during the first admission at our hospital, and AOSD was not suspected at that time. At the second admission, these two typical symptoms of AOSD led us to suspect AOSD.

Joint pain is the second most common symptom of AOSD. The ankles, knees, and wrists are the most commonly involved joints, but the elbows, shoulders and hips have also been described ${ }^{1)}$. Radiographs are usually not helpful in the early stage of the disease (Fig. 2). To our knowledge, there are few reports on the detailed data of joint fluid analysis in AOSD. Fautrel ${ }^{1,6)}$ reported a synovial fluid aspiration of $>2,000$ cells $/ \mathrm{mm} 3$ in AOSD. In this case, the synovial fluid analysis of the knee joint revealed a WBC count of 52,000 cells $/ \mathrm{mm}^{3}$ with a neutrophillic predominance of 94\%, suggesting septic arthritis.

The ESR and CRP are usually positive reflecting the inflammatory nature of this condition. A striking neutrophilic (>80\%) leukocytosis is often seen. Unfortunately, the spiking fever pattern, persistently elevated ESR and CRP, and joint fluid analysis continued to mislead orthopedic surgeons to suspect septic arthritis, which resulted in delayed initiation of appropriate AOSD treatment. Recently, ferritin has been included in diagnostic criteria reflecting its role in oxidative stress ${ }^{9)}$. Overproduction of IL-18 has been related to high serum ferritin levels, which indicate macrophage activation and are suggestive of AOSD $^{1)}$. Ferritin levels represent disease activity and can be used to follow progression or response to the treatment ${ }^{1,6)}$. The high level of ferritin at the diagnosis was normalized after the clinical remission in this case (Fig. 4). Most recently, saturation of glycosylated ferritin mechanism abnormalities has been suggested more specific in ASOD diagnosis, particularly decreased clearance of nonglycosylated ferritin by histiocyte macrophage system ${ }^{9)}$. AOSD can mimic autoimmune disease including rheumatoid arthritis, vasculitis especially polyarteritis nodosa and polymyositis ${ }^{1)}$. Therefore, we tried to exclude autoimmune disease serologic factors including rheumatoid factor (RF), human leukocyte antigen B-27 (HLA-B27), antinuclear antibody (ANA) that showed negative findings (Table
1).

NSAIDs, corticosteroids, and antirheumatic agents are the cornerstones of therapy for AOSD. However, Fautrel ${ }^{1)}$ reported that only $20 \%$ of patients achieved control with NSAIDs. Corticosteroids are usually required to induce clinical remission and the optimal dosage is relatively high (equivalent to 0.5 to $1 \mathrm{mg} / \mathrm{kg} / \mathrm{d}$ of prednisone). Methotrexate appears to be the best choice to control the disease activity and allow for tapering of steroid use in patients with inadequate response to corticosteroids ${ }^{1)}$. Novel agents such as anti-TNF drugs, interleukin blockade, and intravenous gammaglobulin have been tried in small studies with limited success ${ }^{3)}$. In our case, $60 \mathrm{mg}$ of prednisolon was administrated for three weeks, and the clinical remission was rapidly achieved. Then, the dosage was reduced over 3 months in a tapering manner to avoid relapse.

The prognosis of AOSD is usually good, but disseminated intravascular coagulation and multi-organ failure can occur when the diagnosis is delayed and the proper treatment is not performed $^{10)}$.

In conclusion, the chronic articular type of AOSD may be mistaken for septic arthritis as shown in our case. The diagnosis may be delayed because of the clinical and joint fluid analysis findings. We suggest that AOSD should be considered as a diagnosis of exclusion to avoid misdiagnosis with septic arthritis.

\section{References}

1. Fautrel B. Adult-onset Still disease. Best Pract Res Clin Rheumatol. 2008;22:773-92.

2. Yamaguchi M, Ohta A, Tsunematsu T, Kasukawa R, Mizushima Y, Kashiwagi H, Kashiwazaki S, Tanimoto K, Matsumoto Y, Ota T. Preliminary criteria for classification of adult Still's disease. J Rheumatol. 1992;19:424-30.

3. Efthimiou P, Kontzias A, Ward CM, Ogden NS. Adult- onset Still's disease: can recent advances in our understanding of its pathogenesis lead to targeted therapy? Nat Clin Pract Rheumatol. 2007;3:328-35.

4. Still GF. On a form of chronic joint disease in children. Clin Orthop Relat Res. 1990;(259):4-10.

5. Bywaters EG. Still's disease in the adult. Ann Rheum Dis. 1971;30:121-33.

6. Fautrel B. Proposal for a new set of classification criteria of adult-onset still disease. Medicine. 2002;81:194-200.

7. Crispin JC, Martinez-Banos D, Alcocer-Varela J. Adultonset Still disease as the cause of fever of unknown origin. Medicine (Baltimore). 2005;84:331-7. 


\section{Song et al. Adult Onset Still's Disease Misdiagnosed as Septic Arthritis}

8. Mehrpoor G, Owlia MB, Soleimani H, Ayatollahi J. Adultonset Still's disease: a report of 28 cases and review of the literature. Mod Rheumatol. 2008;18:480-5.

9. Fautrel B. Ferritin levels in adult Still's disease: any sugar?
Joint Bone Spine. 2002;69:355-7.

10. Ames PR, Walker E, Aw D, Marshall D, de Villiers F, Staber M. Multi-organ failure in adult onset Still's disease: a septic disguise. Clin Rheumatol. 2009;28(Suppl 1):S3-6. 